\title{
Entry, Descent and Landing Systems Analysis: Exploration Class Simulation Overview and Results
}

\author{
Alicia D. Cianciolo ${ }^{*}$ and Jody L. Davis ${ }^{\dagger}$ \\ NASA Langley Research Center, Hampton, VA 23681 \\ Jeremy D. Shidner ${ }^{\star}$ and Richard W. Powell ${ }^{\S}$ \\ Analytical Mechanics Associates, MS 489, Hampton, VA 23681
}

\begin{abstract}
NASA senior management commissioned the Entry, Descent and Landing Systems Analysis (EDL-SA) Study in 2008 to identify and roadmap the Entry, Descent and Landing (EDL) technology investments that the agency needed to make in order to successfully land large payloads at Mars for both robotic and exploration or human-scale missions. The year one exploration class mission activity considered technologies capable of delivering a 40-mt payload. This paper provides an overview of the exploration class mission study, including technologies considered, models developed and initial simulation results from the EDL-SA year one effort.
\end{abstract}

\section{Nomenclature}

$A D P=$ Advanced Development Program

$A S \quad=$ Aeroshell

$D R A 5=$ Design Reference Architecture 5.0

$E D L-S A=$ Entry, Descent and Landing Systems Analysis

$H I A D=$ Hypersonic Inflatable Aerodynamic Decelerator

$I A D=$ Inflatable Aerodynamic Decelerator

$L / D=$ Lift-to-Drag ratio

$L S A I D=$ Lifting Supersonic Inflatable Aerodynamic Decelerator

$L H I A D=$ Lifting Hypersonic Inflatable Aerodynamic Decelerator

$M O L A=$ Mars Orbiter Laser Altimeter

NTR = Nuclear Thermal Rocket

POST2 = Program to Optimize Simulated Trajectories

SAID = Supersonic Inflatable Aerodynamic Decelerator

TPS = Thermal Protection System

\section{Introduction}

TN July of 2009, NASA released its Human Exploration of Mars Design Reference Architecture 5.0 (DRA5) ${ }^{1}$. The document details the systems and operations required for initial exploration of the planet by humans. Throughout composition of the document, it was apparent that higher fidelity models and advancements in technologies are required to realize the reference architecture. One area that NASA has noted as specifically lacking in technology capability is the Entry, Descent and Landing of "exploration" or human class missions to the surface of the planet.

To that end, the Entry, Descent and Landing System Analysis (EDL-SA) task was initiated to move the initial assessment of the exploration class EDL, outlined in DRA5, to the next level using higher fidelity models and more detailed analysis. The goal of the multi-NASA center three-year task is to identify candidate technologies and evaluate which provide the highest payoffs for future missions. Year one focused on the exploration class missions with landed payloads of approximately $40 \mathrm{mt}$. The focus of year two is to consider two smaller classes of missions;

\footnotetext{
* Aerospace Engineer, Atmospheric Flight and Entry Systems Branch, MS 489, AIAA Senior Member.

${ }^{\dagger}$ Aerospace Engineer, Atmospheric Flight and Entry Systems Branch, MS 489, AIAA Member.

*Aerospace Engineer, Analytical Mechanics Associates, MS 489, AIAA Member.

$\S$ Aerospace Engineer, Analytical Mechanics Associates, MS 489, AIAA Associate Fellow.
}

1

American Institute of Aeronautics and Astronautics 
the one to three metric ton class, called Mars Science Laboratory Improvement class, and a three to five metric ton class, called the Feed Forward mission class. EDL-SA is also tasked, in year three, to create "road maps" that outline proposed development schedules of selected candidate technologies for all mission classes.

Upon completion of the EDL-SA year one task, this paper provides a brief summary of the candidate technologies considered for exploration class missions and the higher fidelity models developed to more accurately characterize the missions. The primary purpose of the paper is to describe the architectures and simulation used to evaluate candidate technologies and to provide a summary of the results of the EDL-SA year one effort.

\section{Candidate Technologies Considered}

EDL-SA study ground rules established that only changes to the Aerocapture and EDL phases of DRA5 were permitted. The commencement of the study included a brainstorming session to identify candidate technologies to be considered. Combinations of the selected technologies formed "architectures". The first, as a direct comparison with DRA5, included a rigid mid range lift-to-drag ratio aeroshell (Rigid Mid-L/D AS) for aerocapture and entry and a second technology, supersonic retro-propulsion, for descent and landing. Other architectures included lifting and drag hypersonic and supersonic inflatable aerodynamic decelerators (HIAD and SIAD respectively) in combination with supersonic or subsonic retro-propulsion. In addition, an all-propulsive architecture was considered for comparison. Table 1 lists the combination of technologies for each of the eight architectures selected for exploration class effort. Figure 1 shows a conceptual illustration of the technology combinations that formed the exploration class mission architectures.

Table 1. Set of Exploration Class Technologies Considered by EDL-SA

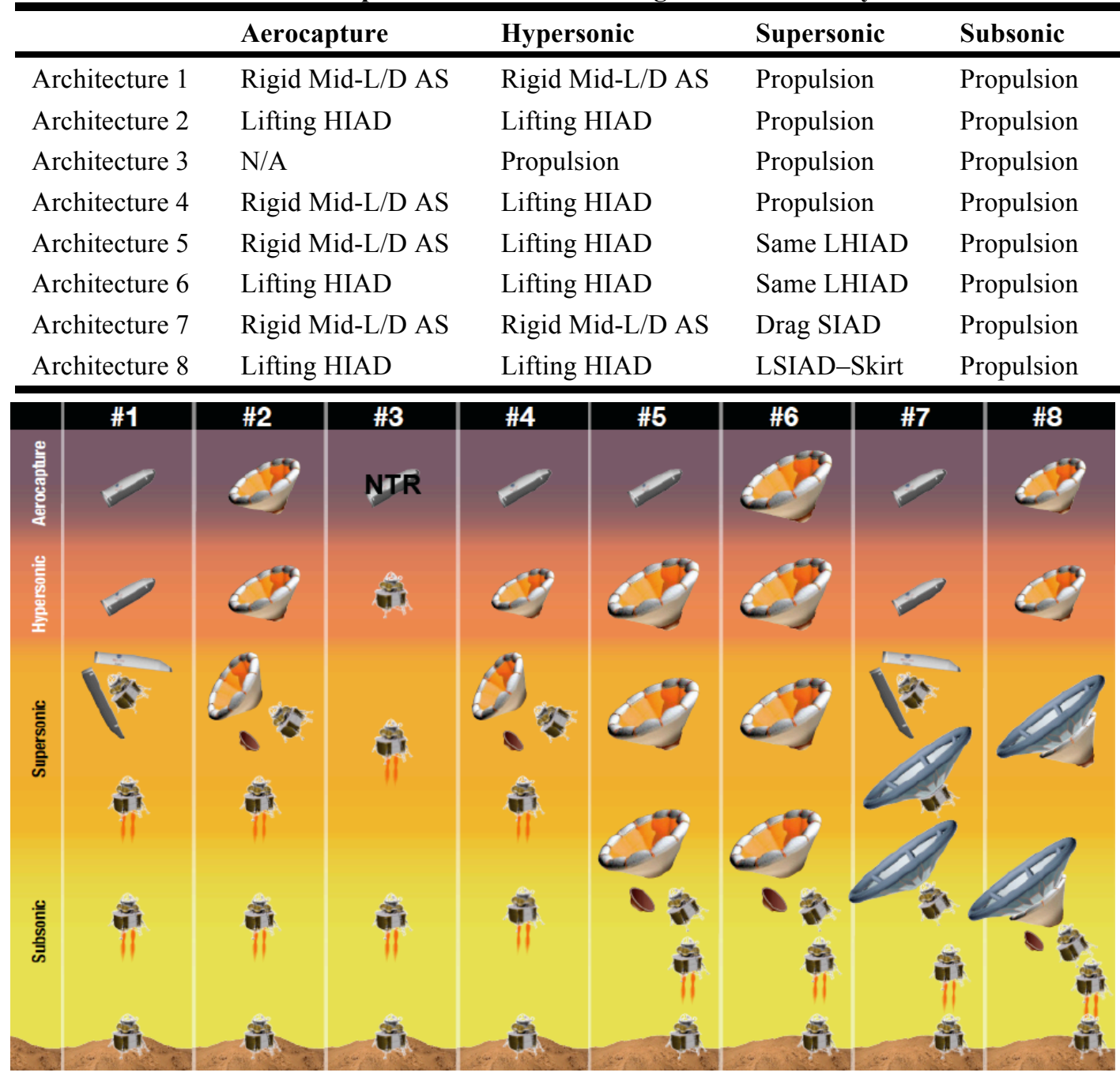

Figure 1. Exploration Class Architectures

2

American Institute of Aeronautics and Astronautics 


\section{The Models and Simulation}

The simulation used to evaluate the EDL-SA architectures was the Program to Optimize Simulated Trajectories $(\mathrm{POST} 2)^{2}$, which is a generalized point mass, discrete parameter targeting and optimization simulation and has extensive heritage for simulating ascent, descent, and orbiting trajectories including past missions like Shuttle, Genesis, Stardust, Genesis, and Mars Missions including, Pathfinder, Odyssey Orbiter, Exploration Rovers, Reconnaissance Orbiter, and the Phoenix Lander. POST2 is also used extensively in current missions like Mars Science Laboratory, Launch Abort Systems and Ares.

Specific models were used to tailor production POST2 for the EDL-SA purposes. Standard Mars atmosphere, gravity, terrain and planet models were used. The bulk of the EDL-SA year one effort was to develop higher fidelity models of low Technology Readiness Level (TRL) components. The details of the individual model development are provided elsewhere ${ }^{3,4,5}$ but a summary is provided here.

\section{A. Mass Model}

The most objective parameter to evaluate and discriminate between architectures is mass, however, there is little mass model precedence for the technologies and sizes of vehicles selected for the EDL-SA exploration class missions. Therefore, mass estimates are based on extrapolations from current studies (i.e. Ares $\mathrm{V}$ for the rigid Mid $\mathrm{L} / \mathrm{D}$ aeroshell) and the use of Response Surface Equations, $\mathrm{RSE}^{5}$. The use of response surfaces make it possible to iterate on a system mass in the POST2 simulation such that the mass is optimized for minimum landed mass to $0 \mathrm{~km}$ above the Mars Orbiter Laser Altimeter (MOLA) defined areoid. Details of the mass model developed for all of the architecture components (HIAD, SIAD, etc) and margins carried can be found in Ref 5. The results of the component masses for each architecture are provided in Table 10.

\section{B. Aerodynamics and Aerothermal Models}

Aerodynamic and aerothermodyanmic modeling was performed for both the Rigid Mid L/D and HIAD configurations using a suite of engineering and high-fidelity aerodynamic and aerothermal analysis tools to develop the aerodynamic and aerothermal databases. The analysis tools including CBAERO ${ }^{7}$, DPLR ${ }^{8,9}$, LAURA $^{10,11}$, NEQAIR ${ }^{12}$, and CART3D ${ }^{13}$. The databases were incorporated into the POST2 simulation to provide drag and heating information critical to trajectory design and the determination of the amount of Thermal Protection System, TPS, required by each configuration. The results also affected the diameter of the drag devices.

\section{Aerocapture and Entry Guidance Algorithms}

Several guidance algorithms were developed and used to design both the aerocapture and EDL portions of flight. The guidance algorithms considered, assessed the feasibility of each architecture to aerocapture with acceptable margin and to maneuver during entry such that the vehicle could reach a specified target. Algorithms considered included the Hybrid Predictor-Corrector Aerocapture Scheme, (HYPAS) ${ }^{14}$, a Numerical Predictor Corrector Guidance (NPC) $)^{15}$, a theoretical "all knowing" guidance and an Apollo derived Analytical Predictor Corrector Guidance(APC) ${ }^{16}$. For entry analysis, the theoretical guidance was used because of its ease of implementation and relatively quick run time. However, to validate the EDL results, entry performance was compared to simulations using both the NPC and APC guidance. Details of the guidance algorithms and their comparative performance as it pertains to EDL-SA can be found in Ref 4.

\section{Supersonic Retro-propulsion Model}

The primary objectives of the Supersonic RetroPropulsion (SRP) study element for EDL were to provide: (a) Isp and thrust/weight ratio guidance for use in higher-level vehicle parametric modeling and (b) high level system design insight to the team. A literature review, commercial modeling software, and system design experience were used to provide these products.

\section{E. Thermal Protection System}

Estimated thermal response models were used in the sizing analysis of the thermal protection systems (TPS) needed for the HIADs and rigid mid-L/D aeroshells used in EDL-SA. For both aeroshells the sizing was performed on a dual heat pulse mission (aerocapture, cool down in orbit and out-of orbit entry). The sizing analysis is based on the tools and practices developed by the Orion TPS Advanced Development Program (ADP). The TPS sizing tool (which makes use of the ablative thermal analysis tool FIAT ${ }^{17}$ ) was extended for EDL-SA to include the capability to size dual layer TPS. A full description of the EDL-SA methodology and results can be found in Ref 18 
In addition, tension cone (SIAD) aerodynamics was based on a previous CEMENT study, all EDL-SA trajectories assume perfect navigation knowledge and that technologies required to perform precise landings will be leveraged from ALHAT.

\section{F. Simulation Setup}

All of the models mentioned above were incorporated into POST2 to provide a means to examine alternative architectures that would provide the aerocapture phase required by DRA5 for the cargo delivery vehicles and the EDL required by both the cargo and crewed vehicles. The approach velocity and target orbit were provided by DRA5. To summarize: 1) the hyperbolic approach velocity was set at $7.36 \mathrm{~km} / \mathrm{s} ; 2$ ) the target orbit was a 1 Sol $(33793 \mathrm{~km} \times 250 \mathrm{~km})$; 3) EDL initiates from the $1 \mathrm{Sol}$ orbit; 4) the landing site is at $0 \mathrm{~m}$ altitude; 5) the touchdown provides $50 \mathrm{~m}$ accuracy; and 6) the deceleration profiles remain within those limits set for a deconditioned crew. It was assumed for all the architectures that a reaction control system (RCS) would be the primary control. To emulate the characteristics of a RCS without having to design a control system, a "pseudo-controller" was used where the bank acceleration, maximum bank rate, and bank direction are modeled.

One simulation was used to evaluate the aerocapture of the Rigid Mid L/D (Architectures 1, 4, 5, 7) and the HIAD (Architecture 2, 6, and 8) into a 1 Sol orbit. A separate simulation, utilizing similar models, was used to design the EDL portion, based on heating information obtained from the aerocapture simulations, to land the $40 \mathrm{mt}$ payload within $50 \mathrm{~m}$ of a specified target.

The first step in the exploration mission EDL design (and the primary difference from DRA5) is to converge the mass model using an in-plane guidance and to determine the appropriate entry point such that the trajectory ends at the preselected landing target. Controlled parameters include the deorbit $\Delta \mathrm{V}$ from apoapsis of a $1 \mathrm{sol}$ orbit and the altitude at which the separation from the drag device occurs such that the vehicle lands within $50 \mathrm{~m}$ of the target. The POST2 simulation modifies the trajectory until parameters such as dynamic pressures and aero-heating characteristics permit mass model convergence.

The first EDL phase, deorbit, begins with the POST2 simulation selecting the deorbit DV from the apoapsis of the 1 sol elliptical orbit to modify heat rate and dynamic pressure as needed to minimize the arrival mass. The second phase, atmospheric entry, begins at a geographic radius of $3255.2 \mathrm{~km}$ and the atmosphere is activated. The third phase, pullout, performed after the mass model has converged, is achieved using the theoretical guidance that employs either a bank reversal strategy based on allowable heading error as a function of velocity, another notable difference from DRA5.

The next phase, heading alignment, begins at a specified velocity; the value may vary between architectures. Bank angle accelerations of five deg $/ \mathrm{s}^{2}$ and a max rate of $20 \mathrm{deg} / \mathrm{s}$ are based in realistic flight controllers. During the heading alignment phase, the objective is to drive the azimuth error to the landing site target to 0 deg at engine initiation. The velocity at which heading alignment begins as well as the allowable errors required to produce three reversals, nominally, may vary between architectures. These phases summarize the entry portion of the trajectory.

The descent begins with the separation phase in which the simulation determines the altitude to jettison the entry drag device (rigid aeroshell, HIAD, or SIAD). For DRA5 the event was assumed to be instantaneous. However, the lack of higher fidelity vehicle separation models and the desire to more realistically model the event, the mass of the decelerator is instantaneously jettisoned and the lander vehicle is allowed to free-fall for a given amount of time to ensure no recontact. Table 2 provides the separation times for each of the architectures based on assumptions made about the nature of the separation. After the allotted time, the terminal descent engines are initiated, commencing the terminal descent phase. Terminal descent design is governed by the desire to balance the fuel saved of using higher throttle settings (therefore higher g's) at a low altitude close to the landing site with maintaining control authority to fly out errors to allow precise landing capability (within $50 \mathrm{~m}$ of the target). Therefore the terminal descent is designed to use, nominally, a maximum throttle setting of $80 \%$ to reach the desired maximum nominal acceleration $(2.5 \mathrm{~g}$ 's) for only an instant before throttling back to fly a linear velocity curve with respect to changes in altitude.

The final phase, landing, also known as the touch down phase, is based on Viking heritage. Once the vehicle reaches $1 \mathrm{~m} / \mathrm{s}$, the velocity is held constant for 5 second until the vehicle touches down at $0 \mathrm{~km}$ above the MOLA areoid at -1.18 deg North latitude and 182 deg East longitude. 
Table 2. Architecture Component Separation Times

\begin{tabular}{clc}
\hline Architecture & \multicolumn{1}{c}{ Separation } & Time (s) \\
\hline 1 & Rigid areoshell from descent stage & 20 \\
$2,4,5,6$ & HIAD from descent stage & 15 \\
7 & Rigid aeroshell from SIAD & 20 \\
7 & SIAD from descent stage & 10 \\
8 & HIAD and SIAD from descent stage & 10 \\
\hline
\end{tabular}

As mentioned previously, certain parameter values like deorbit DV, altitude at separation, heading alignment initiation and azimuth errors, as well as additional separation and deployment events required in Architectures 7 and 8 , can vary between architectures. However, the general EDL strategy remains the same. Figure 2 shows the reference altitude versus velocity plot for each architecture.
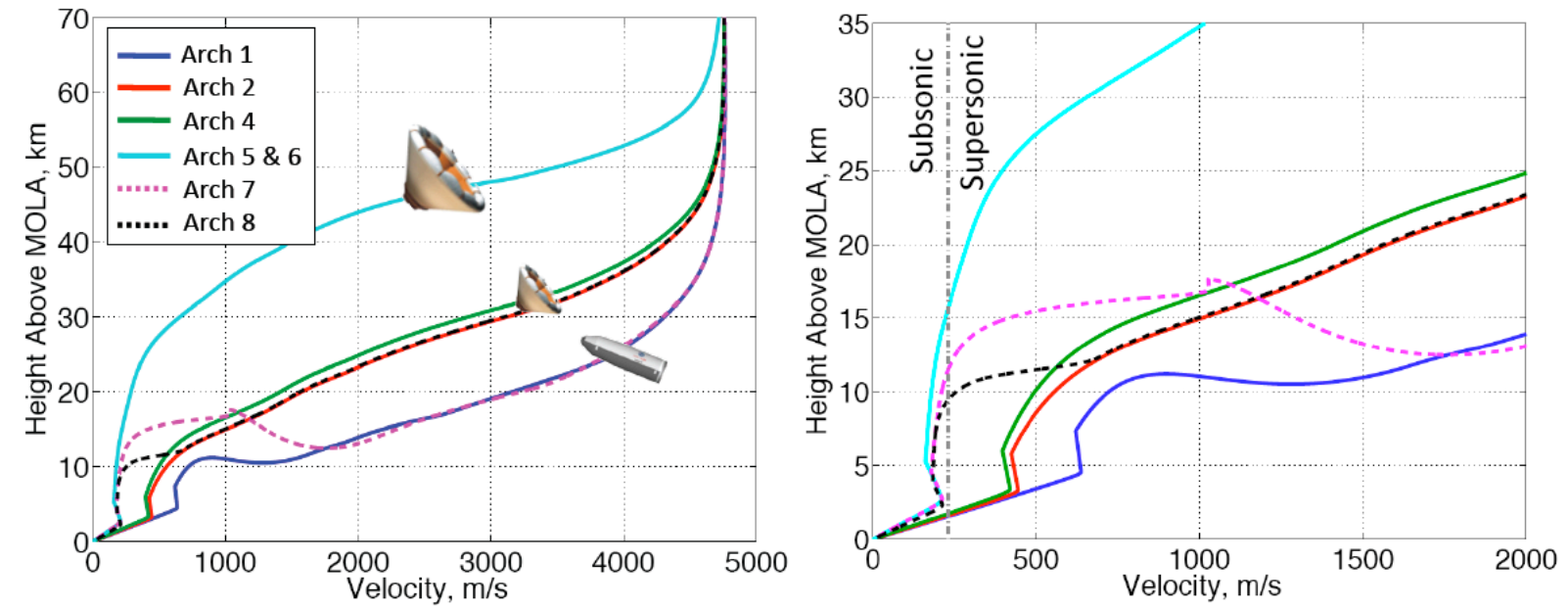

Figure 2. EDL-SA Exploration Class Altitude Versus Velocity

\section{Results}

Each exploration architecture was simulated using POST2 according to the strategy and models outlined in the previous section. Unlike DRA5, Monte Carlo dispersion analysis (using 2000 cases) was preformed on most architectures, including dispersions on aerodynamics, entry state and the atmosphere. Table 3 shows the nominal value and perturbation for each Monte Carlo input parameter. The aerodynamic coefficient multipliers are applied to aerodynamic database output and the atmospheric model (MarsGRAM) dispersions and winds are applied to all cases except the nominal. Detailed nominal trajectory and Monte Carlo results for the architectures are provided below.

Table 3. EDL-SA Monte Carlo Dispersions

\begin{tabular}{llll}
\hline Parameter & Nominal Value & Perturbation & Distribution \\
\hline Nominal Coefficient multiplier & 1 & $+/-10 \%(3 \mathrm{~s})$ & Normal \\
Axial Coefficient multiplier & 1 & $+/-10 \%(3 \mathrm{~s})$ & Normal \\
Angle of Attack (deg) & $55.0,-22.2,-22.2,55.0,-22.2$ & $+/-5(3 \mathrm{~s})$ & Normal \\
Engine I $\mathrm{SP}(\mathrm{sec})$ & 369 & $+/-2.5 \%(3 \mathrm{~s})$ & Normal \\
Entry FPA (deg) & $-10.8,-9.9,-9.0,-10.8,-9.8$ & $+/-0.25(3 \mathrm{~s})$ & Normal \\
Atmospheric Random Number & 1 & $1-29999$ & Uniform \\
Dusttau & 0.7 & $0.1: 0.9$ & Uniform \\
\hline
\end{tabular}




\section{A. Architecture 1}

The Architecture 1 entry configuration, selected for its similarity to DRA5, includes a mid-L/D rigid aeroshell for aerocapture and hypersonic flight and supersonic retro-propulsion for descent and landing. Figure 3 shows the timeline below $30 \mathrm{~km}$. The vehicle flies at 55 degrees angle of attack and has a L/D of 0.5 . The heading alignment phase begins at $1700 \mathrm{~m} / \mathrm{s}$ and the nominal terminal descent throttle setting is set at $80 \%$. However, for the Monte Carlo analysis, the throttle setting is allowed to increase to $90 \%$, which results in several cases that fly a more aggressive descent profile and use considerably less propellant than the nominal case. Additional 1 and 99 percentile Monte Carlo results, including Mach, dynamic pressure and altitude dispersions at aeroshell separation and terminal descent initiation, are included in Table 4. The DRA5 arrival mass estimate of $110.9 \mathrm{mt}$ is remarkable close to the higher fidelity EDL-SA arrival mass of $110.1 \mathrm{mt}$ shown in Table 10, implying that the approximations made for DRA5 adequately represent current knowledge. Because of the use of an aeroshell, Architecture 1 is considered to be at a higher TRL than the drag devices considered in other architectures. However, several technology challenges remain for the aeroshell including packaging, detailed modeling of the separation event and engine initiation at supersonic velocities.

\section{B. Architecture 2}

Architecture 2, which uses a HIAD for aeorcapture and entry along with supersonic retro-propulsion for terminal descent, was selected to evaluate the mass savings of using a dual use TPS HIAD over a rigid mid-L/D areoshell. The $23 \mathrm{~m}$ inflatable design is based on the MIAS concept and is currently at a lower TRL than the rigid aeroshell. The angle of attack for the vehicle is -22 degrees and the $\mathrm{L} / \mathrm{D}$ is 0.33 . Percentile dispersion values are provided in Table 5. The plot of the altitude versus time curve for Architecture 2 is shown in Fig. 4. Plotted for reference is the similar curve for Architecture 1. Note that for Architecture 2 the 3-sigma low range to target at terminal descent initiation has been reduce from $7 \mathrm{~km}$ in Architecture 1 to $2.7 \mathrm{~km}$ resulting in reduced timeline margin for terminal descent. However, use of the HIAD, based on the mass models developed for EDL-SA, reduces the entry mass over Architecture 1 by nearly $25 \mathrm{mt}$, making it an advantageous alternative to Architecture 1, despite technical challenges including HIAD packaging, separation and dual-use TPS.

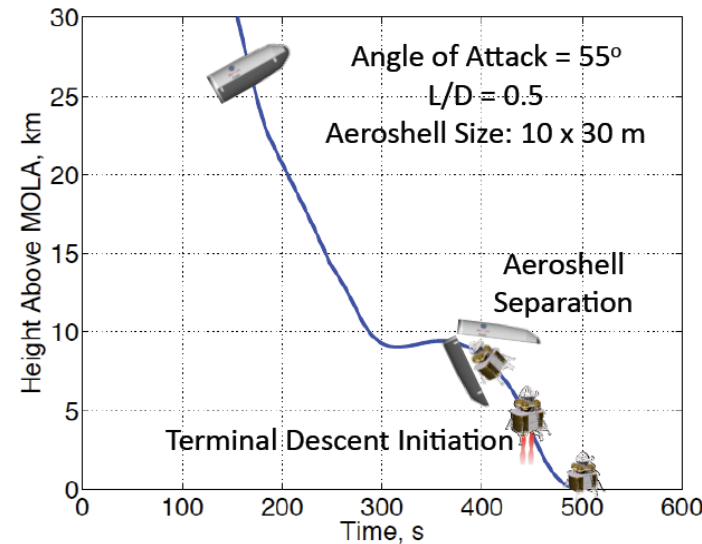

Figure 3. Architecture 1 Altitude vs. Time

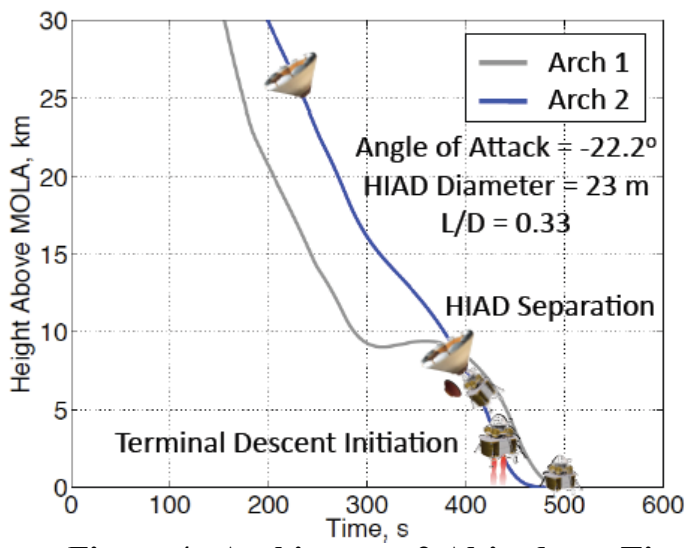

Figure 4. Architecture 2 Altitude vs. Time
Table 4. Architecture 1 Results

\begin{tabular}{lccc}
\hline & $1 \%$ & Mean & $99 \%$ \\
\hline Aeroshell Separation & & & \\
\hline Mach & 2.4 & 2.7 & 3.3 \\
Dyn. Pres., N/m ${ }^{2}$ & 1077.7 & 1283.4 & 1598.3 \\
Altitude, $\mathrm{km}$ & 6.5 & 7.5 & 8.9 \\
Terminal Descent Initiation & & \\
\hline Dyn. Pres., N/m & 1479.2 & 1721.8 & 2049.4 \\
Altitude, km & 3.3 & 4.6 & 6.9 \\
Range To Targ, km & 6.7 & 10.9 & 25.4 \\
Prop Use, $\mathrm{t}$ & 12.4 & 14.2 & 16.9 \\
\hline
\end{tabular}

Table 5. Architecture 2 Results

\begin{tabular}{|c|c|c|c|}
\hline & $1 \%$ & Mean & $99 \%$ \\
\hline \multicolumn{4}{|l|}{ HIAD Separation } \\
\hline Mach & 1.6 & 1.8 & 2.1 \\
\hline Dyn. Pres., N/m² & 563.3 & 646.4 & 780.0 \\
\hline Altitude, km & 5.1 & 5.7 & 6.5 \\
\hline \multicolumn{4}{|c|}{ Terminal Descent Initiation } \\
\hline Dyn. Pres., N/m² & 775.3 & 881.8 & 1027.2 \\
\hline Altitude, km & 2.5 & 3.3 & 4.6 \\
\hline Range To Targ, km & 6.7 & 10.9 & 25.4 \\
\hline Prop Use, t & 12.4 & 14.2 & 16.9 \\
\hline
\end{tabular}

6

American Institute of Aeronautics and Astronautics 


\section{Architecture 3}

Architecture 3, the all-propulsive entry configuration, was the least complex EDL alternative and, using only engines, was considered to be at a relatively high TRL compared to the other architectures. However, there are major technology questions concerning the flowfield interactions of the rocket plume firing into the atmosphere at hypersonic and supersonic conditions. Unknown are the impact of the total pressure on the nozzle performance and internal nozzle flow (possible flow separation), the flow stability and the resulting dynamics on vehicle control, and the resulting drag and aerodynamic heating. Because of the unknowns in drag and aerodynamic heating from the diverted forebody flowfield, the initial study results, used to determine feasibility, included no drag and no aerodynamic heating. Because no estimate of drag was made in this initial assessment, the conservative approach was to assume that no drag would be used to decelerate the system to reduce the $\Delta \mathrm{V}$. The same flow-diversion assumptions were also made for the aerodynamic heating; thus no thermal protection system was used.

Therefore, Architecture 3 assumed that a nuclear thermal rocket, NTR, performs the Mars Insertion burn to place the vehicle into a $1 \mathrm{sol}$ orbit. The strategy for deorbit and entry from $1 \mathrm{Sol}$ was optimized using an instantaneous burn $(\Delta \mathrm{V}=1200 \mathrm{~m} / \mathrm{s})$ to transition to an intermediate orbit of $250 \times 250 \mathrm{~km}$ orbit and then a deorbit burn of $186 \mathrm{~m} / \mathrm{s}$ thereby minimizing the arrival mass and gravity losses.

The trajectory simulation was initiated at the deorbit condition. Initial thrust-to-weight ratio $(\mathrm{T} / \mathrm{W})$ was varied from 1.01 to 3.7 Mars g's, and the altitude at thrust initiation was optimized. At a T/W of 1.010, the thrust initiation altitude was $250 \mathrm{~km}$, and at $\mathrm{T} / \mathrm{W}=3.7$ the altitude was $50 \mathrm{~km}$. With atmospheric interface at approximately 125 $\mathrm{km}$, the cases for T/W greater than 1.8 may not be valid with the no-drag assumption because the nozzle and vehicle forebody are not protected with diverted flow unless a very low-thrust flow divert could be used. A unique EXAMINE based mass model was developed for Architecture 3 and is summarized in Ref. 5 and includes no TPS mass. The deorbit mass results of the trade study can be seen in the solid lines in Fig. 5. No cases exceeded the HSIR requirements for human g levels because the maximum deceleration was 3.7 Mars g's or 1.2 Earth g's. The lowest deorbit mass resulted from a system T/W of 1.01 Mars g's and a total $\Delta \mathrm{V}$ of $5292 \mathrm{~m} / \mathrm{s}$. Using the study baseline LOX/CH4 engines with a specific impulse of 369 seconds, the arrival mass was $310 \mathrm{mt}$ which is $282 \%$ higher than Architecture 1.
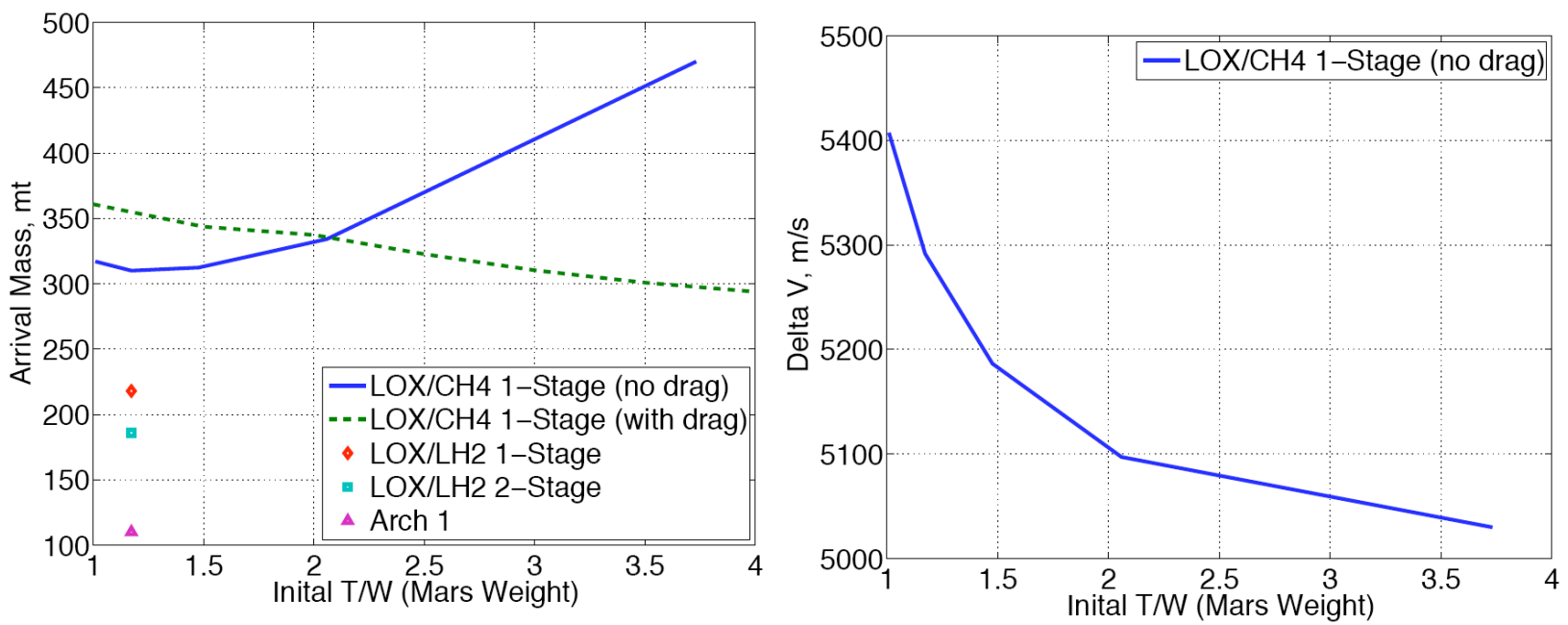

Figure 5. Architecture 3 Arrival Mass and Delta V

The initial study shows that an all-propulsive entry is feasible, albeit with much higher arrival masses than the other architectures considered, and it is noted that the initial study neglected several key parameters. Namely that the drag from recirculization of the flow on the forebody, skin friction drag on the sides, and base drag on the aft body is not negligible and that the no-heating assumption that utilized no TPS may be highly optimistic. Minimal mass margins were carried in this study, which differed from the margin policy used for the other Architectures. Therefore, for comparison, a second study was performed that accounted for the base drag of the aft body assuming a circular area with a nine-meter diameter. The trajectory was designed such that it maintained stagnation heat rates below $25 \mathrm{~W} / \mathrm{cm}^{2}$, which would eliminate the need for TPS. A mass margin policy was included that was similar to that used to evaluate the other Architectures. Also, because atmosphere drag was include, a larger (though not optimized) intermediate orbit of $400 \times 250 \mathrm{~km}$ was considered. As the initial thrust to weight of the vehicle was 
increased, the altitude at which the engines are ignited also drops, resulting in larger losses due to drag. The increases in drag loss result in less propellant required to slow the vehicle and therefore, the arrival mass is reduced. The arrival mass corresponding to the trajectories that include drag loss, thermal constraints and margined masses are shown in the dashed line in Fig. 5. Lower arrival masses can be achieved using larger initial thrust to weight.

The arrival mass can further be reduced by optimizing the intermediate orbit and/or using advanced technology or staging. Using LOX/LH2 engines with a specific impulse of 450 seconds, which are the same technology engines as the cryogenic DRA5 Mars Transfer Vehicle option, the mass may be reduced to $218 \mathrm{mt}$. This option also requires the same zero-boiloff technology as the Earth-to-Mars transfer vehicles. Adding a second LOX/LH2 stage, the mass may be further reduced to $186 \mathrm{mt}$; however, this staging benefit must be traded with the additional cost and reduced reliability of using a second stage with a vehicle that is still $169 \%$ heavier than Architecture 1 . It is noted that there is also no TPS or mass margin included in these estimates. No Monte Carlo analyses were performed for Architecture 3.

\section{Architecture 4}

The entry portion of Architecture 4 is identical to Architecture 2. However, consideration was given to Architecture 4 to compare the mass savings of using a single use (entry only) TPS on a 23 m HIAD to the dual use (aerocapture and entry) version used in Architecture 2. Therefore, different from Architecture 2, Architecture 4 uses a rigid areoshell for aerocapture, then, prior to entry, the HIAD is inflated. The result is a larger arrival mass of $109.0 \mathrm{mt}$ compared to Architecture 2's $83.6 \mathrm{mt}$ but the entry mass is lighter by almost $8 \mathrm{mt}$ due, in part, to the $4 \mathrm{mt}$ lighter HIAD mass. Because of the difference in the entry ballistic coefficient, the architectures have slightly different trajectories but the Monte Carlo analysis results are nearly the same. See Fig. 6 and Table 6. The component mass numbers for the two architectures are compared in Table 10. The increase in launch mass and mission complexity of using a separate aerocapture system, in addition to the technical challenges already present in Architecture 2, did not make this configuration appear practical.

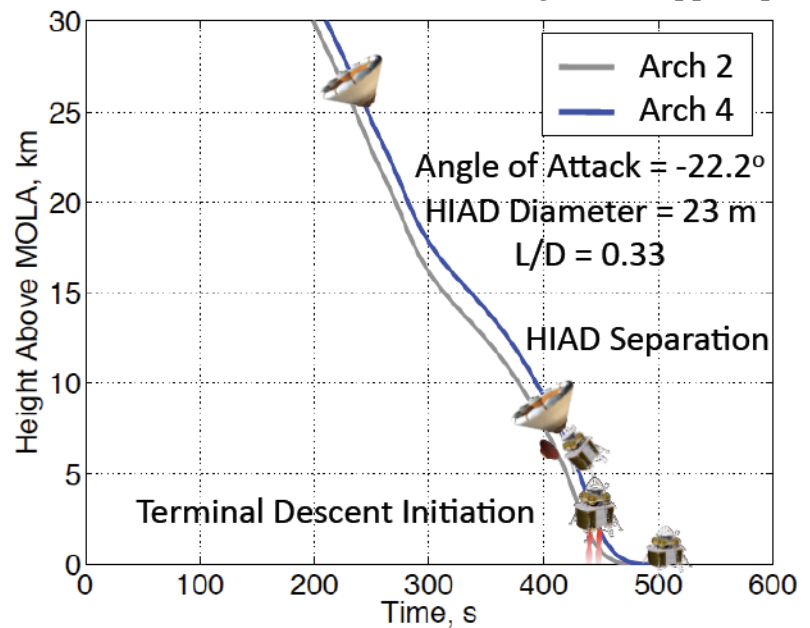

Table 6. Architecture 4 Results

\begin{tabular}{|c|c|c|c|}
\hline & $1 \%$ & Mean & $99 \%$ \\
\hline \multicolumn{4}{|l|}{ HIAD Separation } \\
\hline Mach & 1.5 & 1.7 & 1.9 \\
\hline Dyn. Pres., N/m² & 493.1 & 557.4 & 657.6 \\
\hline Altitude, km & 5.3 & 5.9 & 6.7 \\
\hline \multicolumn{4}{|c|}{ Terminal Descent Initiation } \\
\hline Dyn. Pres., N/m² & 690.5 & 781.1 & 895.9 \\
\hline Altitude, km & 2.7 & 3.3 & 4.5 \\
\hline Range To Targ, km & 3.1 & 4.4 & 7.2 \\
\hline Prop Use, $\mathrm{t}$ & 8.8 & 9.6 & 10.9 \\
\hline
\end{tabular}

Figure 6. Architecture 4 Altitude vs. Time

\section{E. Architectures 5 and 6}

Architectures 5 and 6 were selected to compare the arrival mass savings of using a very large HIAD down to subsonic speeds as an alternative to supersonic retro-propulsion, considered in Architecture 2 and 4. (This is a contingency in the event that supersonic retro-propulsion proved to be an unusable option for an exploration class mission.) Architecture 5, like Architecture 4, compared the mass saving of using a single-use TPS HIAD for entry and a rigid aeroshell for aerocapture. The simulation optimized the HIAD diameters to be $68 \mathrm{~m}$ and $82 \mathrm{~m}$ for Architectures 5 and 6 respectively. While the mass of the aeroshell and smaller HIAD in Architecture 5 did result in a lower arrival mass than Architecture 6's dual use HIAD, there was an issue in the mass model used for both architectures that calls all the results into question. The issue is that the TPS mass model used for the large HIADs was limited to a $50 \mathrm{~m}$ diameter. Extrapolation beyond that diameter resulted in the assumption that the areal density of the material was constant, which is not likely to be the case. Also there are additional EDL timeline challenges of successfully slowing the vehicle to subsonic speeds with adequate altitude margin to initiate the terminal descent engines and land at the target. The timeline for Architecture 6 is provided in Fig. 7. Architecture 5 is very similar.

8

American Institute of Aeronautics and Astronautics 
A low throttle setting of nominally $65 \%$ is used to slow the vehicle starting at approximately $2.5 \mathrm{~km}$ such that the vehicle can touch down at the target. The large diameter HIAD flies at the same angle of attack -22 deg and same $\mathrm{L} / \mathrm{D}$ as in Architecture 2. Monte Carlo one and $99 \%$ dispersions are also provided in Table 7. However, the results indicate that investments in supersonic retro-propulsion might be a more prudent choice over very large diameter HIAD systems, which will have packaging and separation technical challenges.

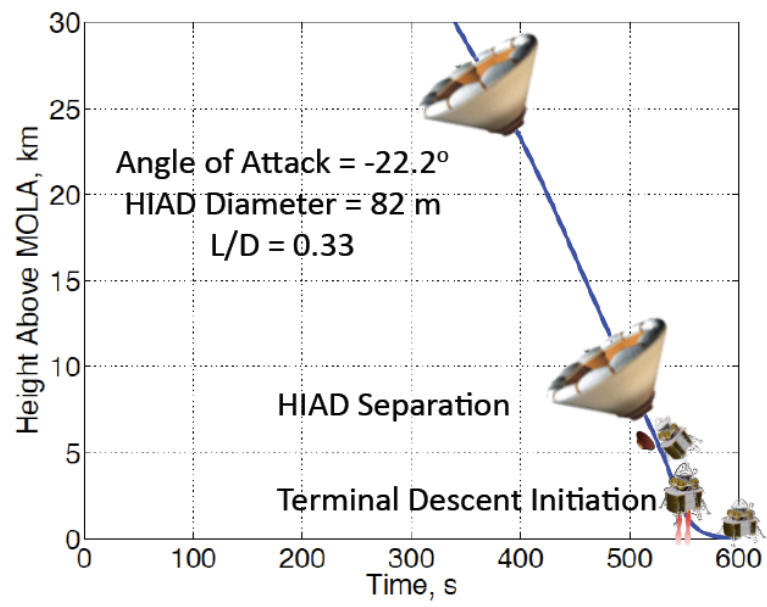

Table 7. Architecture 6 Results

\begin{tabular}{lccc}
\hline & $1 \%$ & Mean & $99 \%$ \\
\hline HIAD Separation & \multicolumn{3}{c}{} \\
\hline Mach & 0.7 & 0.7 & 0.7 \\
Dyn. Pres., N/m ${ }^{2}$ & 93.3 & 99.5 & 106.3 \\
Altitude, km & 5.0 & 5.2 & 5.4 \\
Terminal Descent Initiation & & \\
\hline Dyn. Pres., N/m & & & \\
Altitude, km & 199.9 & 214.1 & 230.4 \\
Range To Targ, km & 2.6 & 2.7 & 2.9 \\
Prop Use, $\mathrm{t}$ & 0.6 & 0.7 & 1.0 \\
& 6.7 & 6.9 & 7.2 \\
\hline
\end{tabular}

Figure 7. Architecture 6 Altidue vs. Time

\section{F. Architecture 7}

Architecture 7 was selected as an alternative to Architecture 1 assuming that supersonic retro-propulsion was an infeasible option for exploration-class missions. Architecture 7 replaces supersonic retro-propulsion with a $51 \mathrm{~m}$ diameter SIAD and subsonic retro-propulsion. The nominal timeline compared to Architecture 1 is shown in Fig. 8. Monte Carlo statistics are provided in Table 8 . The entry strategy remains the same. To accommodate wind effects on the large unguided SIAD, a cross range offset at entry was included in the simulation. The altitude at engine initiation was also maximized to account for the winds during the unguided portion of the trajectory. Therefore, the maximum throttle setting during terminal decent was reduced from 80 to $65 \%$, as well as the system thrust-to-weight from 3.0 to $2.5 \mathrm{~g}$ 's. In addition to the aeroshell packaging and separation technical challenges, Architecture 7 has SIAD packaging, deployment, inflation and separation technical challenges. In the end, the added complexity of EDL for Architecture 7, in particular the unguided portion of the trajectory on the SIAD, results in a mass savings of only $3 \mathrm{mt}$ of Mars arrival mass compared to Architecture 1.

Table 8. Architecture 7 Results

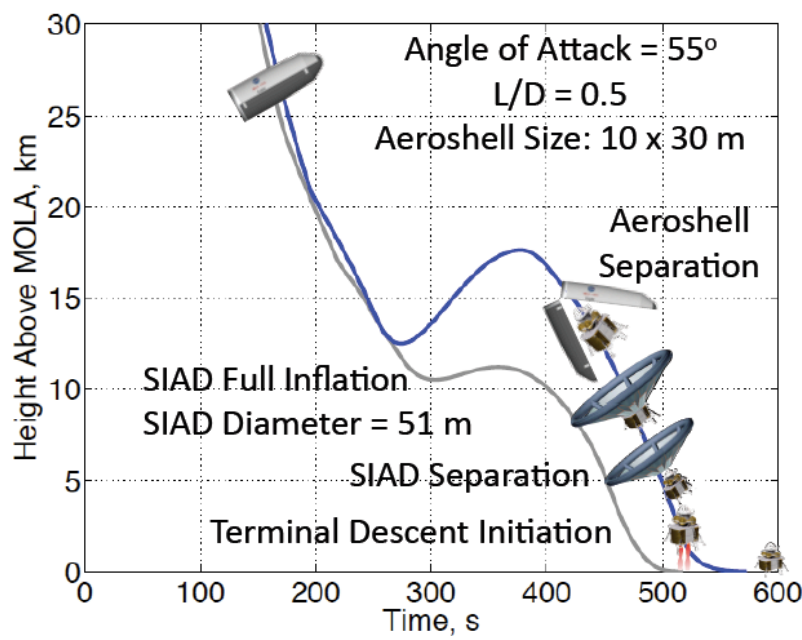

Figure 8. Architecture 7 Altitude vs. Time

\begin{tabular}{lccc}
\hline & $1 \%$ & Mean & $99 \%$ \\
\hline Aeroshell Separation & \multicolumn{3}{c}{} \\
\hline Mach & 0.7 & 0.7 & 0.8 \\
Dyn. Pres., N/m ${ }^{2}$ & 115.3 & 125.3 & 133.6 \\
Altitude, km & 4.3 & 4.5 & 4.6 \\
SIAD Full Inflation & \multicolumn{3}{c}{} \\
\hline Mach & 3.5 & 3.8 & 4.1 \\
Dyn. Pres., N/m ${ }^{2}$ & 983.6 & 1151.0 & 1406.1 \\
Altitude, km & 12.2 & 16.0 & 18.5 \\
SIAD Separation & & & \\
\hline Mach & 4.4 & 4.6 & 4.8 \\
Dyn. Pres., N/m ${ }^{2}$ & 1096.7 & 1416.6 & 2188.1 \\
Altitude, km & 12.8 & 17.5 & 20.3 \\
Terminal Descent Initiation & & \\
\hline Dyn. Pres., N/m ${ }^{2}$ & 190.3 & 209.8 & 225.4 \\
Altitude, km & 2.6 & 2.7 & 2.8 \\
Range To Targ, km & 0.0 & 0.5 & 1.5 \\
Prop Use, t & 6.9 & 7.4 & 8.2 \\
\hline
\end{tabular}

9

American Institute of Aeronautics and Astronautics 


\section{G. Architecture 8}

Architecture 8 considered a HIAD plus SIAD configuration as an alternative to a single large HIAD from Architecture 6 to reduce the vehicle to subsonic speeds prior to engine initiation. The HIAD/SIAD combination included the same $23 \mathrm{~m}$ HIAD used in Architecture 2 and 4. The simulation determined the size of the SIAD (44 m), and it was deployed nominally at Mach 2.6, such that the terminal descent engine initiation would occur subsonicly. The inflatable designs were based on the MIAS and IRDT studies. The advantage of a SIAD is the lighter mass due to deployment after peak heating, which eliminates the need for TPS, while still providing drag to slow the vehicle to subsonic speeds at engine initiation. To compare the mass savings of a HIAD/SIAD system to a larger HIAD only system, the results of the mass model suggest that using a SIAD can save approximately $40 \mathrm{mt}$ of HIAD mass while costing only approximately $2 \mathrm{mt}$ in SIAD mass for a net savings in Architecture 8 of approximately $38 \mathrm{mt}$ over Architecture 6 as shown in Table 10. However note the caveat of extrapolating HIAD diameter used in Architecture 6. The mass savings in reality may not be as significant. However, comparing use of a HIAD/SIAD combination to using supersonic retro-propulsion in Architecture 2, the use of a SIAD only saves approximately 3 $\mathrm{mt}$ of arrival mass. Despite the mass advantages, combinations of inflatables are arguably at a lower TRL than single inflatable structures, and in this particular configuration, also has additional technical challenges associated with the SIAD, increased complexity of EDL and a compressed the EDL timeline. See Fig. 9 and Table 9.

Table 9. Architecture 8 Results

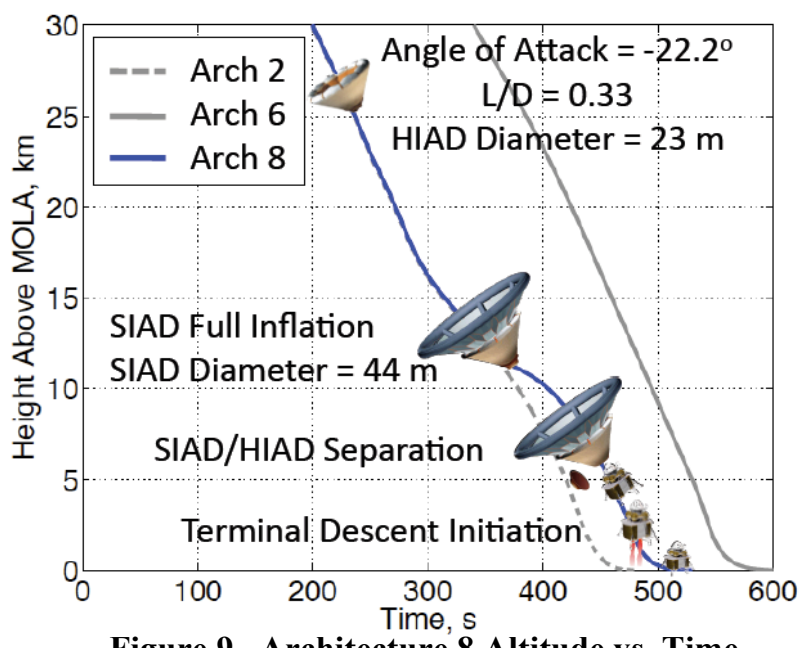

Figure 9. Architecture 8 Altitude vs. Time

\begin{tabular}{|c|c|c|c|}
\hline & $1 \%$ & Mean & $99 \%$ \\
\hline \multicolumn{4}{|l|}{ Aeroshell Separation } \\
\hline $\begin{array}{l}\text { Mach } \\
\text { Dyn. Pres., N/m² } \\
\text { Altitude, km } \\
\text { SIAD Full Inflation }\end{array}$ & $\begin{array}{c}0.7 \\
131.5 \\
3.7\end{array}$ & $\begin{array}{c}0.8 \\
144.1 \\
3.9\end{array}$ & $\begin{array}{c}0.8 \\
155.6 \\
4.0\end{array}$ \\
\hline $\begin{array}{l}\text { Mach } \\
\text { Dyn. Pres., N/m² } \\
\text { Altitude, } \mathrm{km} \\
\text { SIAD Separation }\end{array}$ & $\begin{array}{c}2.4 \\
654.7 \\
8.4\end{array}$ & $\begin{array}{c}2.6 \\
775.7 \\
11.9\end{array}$ & $\begin{array}{c}2.7 \\
958.4 \\
14.3\end{array}$ \\
\hline $\begin{array}{l}\text { Mach } \\
\text { Dyn. Pres., N/m² } \\
\text { Altitude, km } \\
\text { Terminal Descent Init }\end{array}$ & $\begin{array}{c}2.9 \\
804.6 \\
8.9 \\
\end{array}$ & $\begin{array}{c}3.0 \\
1008.0 \\
12.5\end{array}$ & $\begin{array}{c}3.2 \\
1379.0 \\
15.0\end{array}$ \\
\hline $\begin{array}{l}\text { Dyn. Pres., N/m² } \\
\text { Altitude, } \mathrm{km} \\
\text { Range To Targ, km } \\
\text { Prop Use, } \mathrm{t}\end{array}$ & $\begin{array}{l}195.8 \\
2.2 \\
1.0 \\
6.2\end{array}$ & $\begin{array}{l}222.0 \\
2.3 \\
1.1 \\
6.4\end{array}$ & $\begin{array}{c}242.6 \\
2.5 \\
1.5 \\
6.7\end{array}$ \\
\hline
\end{tabular}

A note regarding the mass model developed for EDL-SA. It is acknowledged that there are many cost functions by which to evaluate the various technologies and Architectures considered for this study. However, any method of evaluation will contain high levels of uncertainty due to the fact that systems being evaluated have few points of comparison. For example, it is difficult to quantify timeline margin when the details of transitions are only now being investigated. It is difficult to apply error margins on the mass model because there are few data points with which to validate the model. However, mass is the easiest to quantify. For this reason, the primary discriminator between architectures has been component masses that include standard 30\% mass margins and as well as a $15 \%$ mass growth allowance. The component values are provided for each Architecture in Table 10. It is also recognized that environmental parameters are of interest for defining and identifying requirements for various technologies. For that reason Table 11 has been included.

\section{Conclusion}

The goal of the multi-NASA center three-year task is to identify candidate technologies and evaluate which provide the highest payoffs for future missions. The primary purpose of the paper is to describe the architectures and simulation used to evaluate candidate technologies and to provide a summary of the results of the EDL-SA year one effort. In summary, the impracticality of designing and carrying separate systems for aerocapture and entry has rendered the Architecture 4 and 5 unlikely candidates for further study. Decreasing engine throttle settings and 
igniting engines at higher altitudes has mitigated the small range-to-targets at subsonic engine initiation in Architectures 6,7 and 8. The more significant issue is accurate mass modeling of large HIADs ( $>50 \mathrm{~m}$ diameter). The assumptions made in this study, namely to assume constant areal density for diameters larger than $50 \mathrm{~m}$ is probably too conservative. Current mass models perhaps unrealistically penalize those architectures, namely 5 and 6 , although packaging and inflation issues remain. The compressed EDL timeline that results from using subsonic retro-propulsion make Architectures 6, 7, and 8 less likely candidates for exploration-class missions but should not be ruled out for robotic missions. Finally, Architecture 1 and 2, with their relatively less complex EDL, indicate that the HIAD, mid L/D aeroshell and supersonic retro-propulsion are technologies to be considered in more detailed study for exploration class missions.

Table 10. Component Dimensions and Masses using Standard Margins

\begin{tabular}{|c|c|c|c|c|c|c|c|c|}
\hline Architecture & 1 & 2 & 3 & 4 & 5 & 6 & 7 & 8 \\
\hline $\begin{array}{l}\text { HIAD Diameter }(\mathrm{m}) \\
\text { Aerocapture/Entry }\end{array}$ & -- & 23.0 & -- & 23.0 & 67.8 & 81.9 & -- & 23.0 \\
\hline SIAD Diameter (m) & -- & -- & -- & -- & -- & -- & 44.6 & 44.3 \\
\hline $\begin{array}{l}\text { Aerocapture/Entry BN } \\
\left(\mathrm{kg} / \mathrm{m}^{2}\right)\end{array}$ & 396.1 & 154.0 & -- & 134.6 & 20.6 & 20.4 & 412.0 & 152.5 \\
\hline Descent BN $\left(\mathrm{kg} / \mathrm{m}^{2}\right)$ & -- & -- & -- & -- & -- & -- & 27.2 & 40.8 \\
\hline \multicolumn{9}{|c|}{ Mass (mt) } \\
\hline Arrival Mass & 110.1 & 83.6 & 265.2 & 109.0 & 133.5 & 140.5 & 107.4 & 80.6 \\
\hline Deorbit Mass & 109.2 & 82.8 & 188.1 & 75.1 & 98.0 & 139.4 & 106.3 & 79.6 \\
\hline Aeroshell, AS (Total) & 28.9 & 0.0 & -- & 26.0 & 27.0 & 0.0 & 28.8 & 0.0 \\
\hline AS Structure & 18.3 & -- & -- & 18.4 & 19.3 & -- & 18.2 & -- \\
\hline AS TPS & 10.6 & -- & -- & 7.6 & 7.6 & -- & 10.6 & -- \\
\hline $\begin{array}{l}\text { Avionics and Separation } \\
\text { Structure }\end{array}$ & 2.0 & 2.1 & -- & 4.3 & 7.4 & 8.5 & 2.0 & 1.9 \\
\hline Entry RCS (Total) & 10.8 & 7.1 & -- & 9.9 & 14.7 & 16.8 & 11.7 & 7.4 \\
\hline RCS Dry Mass & 5.2 & 2.7 & -- & 5.8 & 9.2 & 9.9 & 6.1 & 2.9 \\
\hline RCS Propellant & 5.5 & 4.4 & & 4.1 & 5.5 & 6.9 & 5.6 & 4.5 \\
\hline HIAD (Total) & -- & 10.6 & -- & 6.0 & 25.7 & 56.0 & -- & 10.6 \\
\hline HIAD Structure & -- & 6.0 & -- & 3.1 & 10.7 & 22.3 & -- & 5.9 \\
\hline HIAD TPS & -- & 4.7 & -- & 2.9 & 15.0 & 33.7 & -- & 4.7 \\
\hline SIAD Mass & -- & -- & -- & -- & -- & -- & 6.8 & 2.1 \\
\hline Descent Stage, DS & 28.4 & 23.8 & 148.1 & 22.8 & 18.7 & 19.2 & 18.1 & 18.6 \\
\hline DS Dry Mass & 12.3 & 11.7 & 19.2 & 11.2 & 9.9 & 10.5 & 10.2 & 10.4 \\
\hline DS Propellant & 16.2 & 12.0 & 128.9 & 11.6 & 8.8 & 8.7 & 7.9 & 8.2 \\
\hline Landed Mass & 52.3 & 51.8 & 74.7 & 51.2 & 49.9 & 50.5 & 50.2 & 50.4 \\
\hline Payload Mass & 40.0 & 40.0 & 40.0 & 40.0 & 40.0 & 40.0 & 40.0 & 40.0 \\
\hline
\end{tabular}

11

American Institute of Aeronautics and Astronautics 
Table 11. Nominal Environment Conditions

\begin{tabular}{|c|c|c|c|c|c|c|c|c|}
\hline Architecture & 1 & 2 & 3 & 4 & 5 & 6 & 7 & 8 \\
\hline \multicolumn{9}{|c|}{ Aerocapture } \\
\hline $\begin{array}{l}\text { Peak Stag Heat } \\
\text { Rate }\left(\mathrm{W} / \mathrm{cm}^{2}\right)\end{array}$ & 122.8 & 74.6 & -- & -- & -- & 36.6 & -- & -- \\
\hline $\begin{array}{l}\text { Peak Ames Heat } \\
\text { Rate }\left(\mathrm{W} / \mathrm{cm}^{2}\right)\end{array}$ & 335.8 & 88.8 & -- & -- & -- & 36.7 & -- & -- \\
\hline $\begin{array}{l}\text { Total Stag Heat } \\
\text { Load }\left(\mathrm{J} / \mathrm{cm}^{2}\right)\end{array}$ & 13563.8 & 7665.3 & -- & -- & -- & 3894.9 & -- & -- \\
\hline $\begin{array}{l}\text { Total Ames Heat } \\
\operatorname{Load}\left(\mathrm{J} / \mathrm{cm}^{2}\right)\end{array}$ & 34499.4 & 7944.4 & -- & -- & -- & 3433.4 & -- & -- \\
\hline $\begin{array}{l}\text { Peak Dynamic Pres. } \\
\left(\mathrm{N} / \mathrm{m}^{2}\right)\end{array}$ & 10600.9 & 4072.6 & -- & -- & -- & 1061.6 & -- & -- \\
\hline Peak Earth G load & 3.0 & 2.9 & -- & -- & -- & 3.0 & -- & -- \\
\hline \multicolumn{9}{|c|}{ Entry } \\
\hline Deorbit DV (m/s) & 15.3 & 14.0 & 1307.7 & 13.9 & 12.6 & 12.6 & 15.1 & 14.0 \\
\hline $\begin{array}{l}\text { Peak Stag Heat } \\
\text { Rate }\left(\mathrm{W} / \mathrm{cm}^{2}\right)\end{array}$ & 19.7 & 27.3 & 0.7 & 25.6 & 9.9 & 9.9 & 19.5 & 27.2 \\
\hline $\begin{array}{l}\text { Peak Ames Heat } \\
\text { Rate }\left(\mathrm{W} / \mathrm{cm}^{2}\right)\end{array}$ & 119.5 & 27.0 & 5.0 & 24.4 & 6.8 & 5.2 & 117.9 & 26.8 \\
\hline $\begin{array}{l}\text { Total Stag Heat } \\
\text { Load }\left(\mathrm{J} / \mathrm{cm}^{2}\right)\end{array}$ & 2270.7 & 3070.5 & 102.6 & 2875.6 & 1149.5 & 1143.8 & 2363.6 & 3047.4 \\
\hline $\begin{array}{l}\text { Total Ames Heat } \\
\text { Load }\left(\mathrm{J} / \mathrm{cm}^{2}\right)\end{array}$ & 13297.4 & 2610.0 & 661.9 & 2403.1 & 723.4 & 547.7 & 13833.8 & 2593.1 \\
\hline $\begin{array}{l}\text { Peak Dynamic Pres. } \\
\left(\mathrm{N} / \mathrm{m}^{2}\right)\end{array}$ & 10506.5 & 4240.1 & 1823.1 & 3706.2 & 572.4 & 569.2 & 10961.2 & 4194.5 \\
\hline Peak Earth G load & 3.0 & 3.0 & 2.0 & 3.0 & 3.0 & 3.0 & 5.2 & 3.0 \\
\hline Descent DV (m/s) & 797.8 & 601.0 & 3701.5 & 585.9 & 447.1 & 435.8 & 438.2 & 406.4 \\
\hline
\end{tabular}

\section{References}

${ }^{1}$ Drake, B. G., (ed), "Human Exploration of Mars Design Reference Architecture 5.0," s.1. : NASA-SP-2009-566, July 2009

${ }^{2}$ Powell, R. W., et al., "Program to Optimize Simulated Trajectories (POST2), Vol. II Utilization Manual." Version 1.1.1G, May 2000.

${ }^{3}$ Zumwalt, C. H., Sostaric, R. R., Westhelle, C. H., and Cianciolo, A. D., “Aerocapture Guidance and Performance at Mars for High Mass Systems” AIAA/AAS Astrodynamics Specialist Conference, Toronto, Canada, August 2010.

12

American Institute of Aeronautics and Astronautics 
${ }^{4}$ Davis J. L., Cianciolo, A. D., Llama, E. G., Shidner, J. D. and Powell, R. W., "Guidance and Control Algorithms for the Mars Entry Descent and Landing Systems Analysis," AIAA/AAS Astrodynamics Specialist Conference, Toronto, Canada, August 2010.

${ }^{5}$ Samareh, J. A. and Komar D. R., “ Parametric Mass Modeling for Mars Entry, Descent, and Landing System Analysis Study," 49th AIAA Aerospace Sciences Meeting in Orlando, FL, January 4-7, 2011

${ }^{6}$ Zang, T. A. et al. "Overview of the NASA Entry, Descent and Landing Systems Analysis Study." Space 2010 Conference.

${ }^{7}$ Kinney, D.J., "Aero-Thermodynamics for Conceptual Design”, AIAA-2004-13382, January 2004.

${ }^{8}$ Wright, M., Loomis, M., and Papadopoulos, P., "Aerothermal Analysis of the Project Fire II Afterbody Flow," Journal of Thermophysics and Heat Transfer, Vol. 17, No. 2, 2003, pp. 240-249.

${ }^{9}$ Wright, M., Candler, G., and Bose, D., "Data-Parallel Line Relaxation Method for the Navier-Stokes Equations," AIAA Journal, Vol. 36, No. 9, 1998, pp. 1603-1609.

${ }^{10}$ Gnoffo, P. A., Gupta, R. N., and Shinn, J., "Conservation Equations and Physical Models for Hypersonic Air Flows in Thermal and Chemical Nonequilibrium," NASA TP 2867, 1989.

${ }^{11}$ Gnoffo, P. A., "An Upwind-Biased, Point-Implicit Relaxation Algorithm for Viscous, Compressible Perfect-Gas Flows," NASA TP 2953, 1990.

${ }^{12}$ Whiting, E.E., Yen, L., Arnold, J. O., and Paterson, J.A., "NEQAIR96”, Nonequilibrium and Equilibrium Radiative Transport and Spectra Program: User's Manual," NASA RP-1389, Dec. 1996.

${ }^{13}$ Aftosmis, M., "3D Applications of Cartesion Grid Euler Method." AIAA 95-0853

${ }^{14}$ Masciarelli, J., Rousseau, S., Fraysse, H., and Perot, E. "An Analytic Aerocapture Guidance Algorithm for the Mars Sample Return Orbiter," AIAA-2000-4116, 2000.

${ }^{15}$ Powell, Richard W., "Numerical Roll Reversal Predictor Corrector Aerocapture and Precision Landing Guidance Algorithms for the Mars Surveyor Program 2001 Missions," AIAA 1998.

${ }^{16}$ Moseley, P. E., "The Apollo Entry Guidance: A Review of the Mathematical Development and Its Operational Characteristics," TRW Note No. 69-FMT-791, TRW, December 1, 1969.

${ }^{17}$ Chen, Y. K. and Milos, F. S., "Ablation and Thermal Response Program for Spacecraft Heatshield Analysis," Journal of Spacecraft and Rockets, Vol. 36, No. 3, 1999, Pp. 475-483. 